\title{
Dynamical Theory of X-Ray Diffraction
}

\author{
ANDRÉ AUTHIER \\ Université P. et M. Curie, Paris
}




\section{Contents}

I Background and basic results

1 Historical developments 3

$\begin{array}{lll}1.1 & \text { Prologue } & 3\end{array}$

1.2 The discovery of X-ray diffraction 4

1.3 The geometrical theory of diffraction 5

1.4 Darwin's dynamical theory of diffraction 6

$\begin{array}{lll}1.5 & \text { Extinction theories } & 8\end{array}$

1.6 Ewald's dynamical theory 11

1.7 Early confirmations of the dynamical theory 13

$\begin{array}{lll}1.8 & \text { Laue's dynamical theory } & 14\end{array}$

1.9 Umweganregung and Aufhellung $\quad 14$

1.10 The properties of wavefields 16

1.10.1 Anomalous absorption (the Borrmann effect) 16

1.10.2 Wavefield trajectories 20

1.10.3 Pendellösung 23

1.11 Diffraction by deformed crystals $\quad 25$

1.12 Modern times 26

2 Properties of the electromagnetic field-propagation and scattering

2.1 Maxwell's equations 28

2.2 The electrodynamic potentials in vacuum 29

2.2.1 The vector and scalar potentials $\quad 29$

2.2.2 The retarded potentials 30

2.3 The electrodynamic potentials in polarized media 31

2.4 Hertz vectors (polarization potentials) 31

2.5 Propagation of an electromagnetic wave in vacuum 33

$\begin{array}{lll}2.6 & \text { Scattering of X-rays by an electron } & 33\end{array}$

2.7 Polarizability of matter for X-rays 36

2.7.1 Elementary dispersion theory $\quad 36$

2.7.2 Fourier expansion of the polarizability 37

2.7.3 Index of refraction 41

2.7.4 Absorption 41

2.8 Ewald's dispersion theory 43

2.9 Propagation equation of an electromagnetic wave in materials in Laue's dynamical theory 49

2.9.1 Laue's basic assumption 49 


\subsubsection{Propagation equation}

2.10 Specular reflection-Fresnel relations

3 Geometrical theory of X-ray diffraction

3.1 Classical scattering by an electron-polarization $\quad 57$

3.2 Amplitude diffracted by a periodic electron distribution 58

$\begin{array}{lll}3.3 & \text { Intensity diffracted by a small crystal } & 61\end{array}$

$\begin{array}{lll}3.4 & \text { Reflectivity } & 63\end{array}$

3.5 Integrated intensity $\quad 65$

$\begin{array}{lll}3.6 & \text { Mosaic crystals } & 67\end{array}$

$4 \quad$ Elementary dynamical theory $\quad 68$

$4.1 \quad$ Limitations of the geometrical theory 68

4.2 Introduction of the dispersion surface 69

$\begin{array}{lll}4.3 & \text { Analogy with the band theory of solids } & 71\end{array}$

$\begin{array}{lll}4.4 & \text { Propagation equation } & 73\end{array}$

4.5 Fundamental equations of dynamical theory 74

4.6 Amplitude ratio of the refracted and reflected waves 79

4.7 Solutions of plane-wave dynamical theory 80

4.7.1 Boundary conditions $\quad 80$

4.7.2 Departure from Bragg's angle of the incident wave 81

$\begin{array}{lll}\text { 4.7.3 Transmission and reflection geometries } & 82\end{array}$

$\begin{array}{lll}\text { 4.7.4 Deviation parameter } & 85\end{array}$

4.7.5 Determination of the tiepoints 85

$\begin{array}{lll}\text { 4.7.6 Effective absorption coefficient } & 87\end{array}$

4.8 The diffracted waves in the transmission geometry 88

4.8.1 Double refraction 88

4.8.2 Boundary conditions for the amplitudes at the entrance surface $\quad 88$

$\begin{array}{lll}\text { 4.8.3 Intensities of the reflected and refracted waves } & 89\end{array}$

4.8.4 Anomalous absorption 90

$\begin{array}{ll}\text { 4.8.5 Boundary conditions at the exit surface } & 92\end{array}$

$\begin{array}{lll}4.8 .6 & \text { Reflectivity } & 94\end{array}$

4.8.7 Pendellösung 96

$\begin{array}{lll}4.8 .8 & \text { Integrated intensity } & 98\end{array}$

4.9 The diffracted waves in the reflection geometry 99

$\begin{array}{lll}4.9 .1 & \text { Tiepoints } & 99\end{array}$

4.9.2 Thick crystals - total reflection $\quad 99$

4.9.3 Thin crystals 102

4.10 Influence of the asymmetry on the position and width of the rocking curve and of the angular distribution of the reflected beam

4.11 Comparison with geometrical theory 107

4.12 Dynamical diffraction by quasicrystals 110 
5 Properties of wavefields

5.1 Relations between the field vectors 115

$\begin{array}{lll}5.2 & \text { Fundamental equations of the dynamical theory } & 117\end{array}$

5.3 The dispersion equation in the two-beam case 118

$\begin{array}{lll}5.4 & \text { Poynting vector of the wavefields } & 121\end{array}$

5.5 Determination of the tiepoints-geometrical interpretation of the deviation parameter

5.5.1 Boundary condition for the wavevectors

5.5.2 Deviation from Bragg's angle of the middle of the reflection domain

5.5.3 Coordinates of the tiepoint

5.5.4 Deviation parameter, Pendellösung distance and Darwin width in the transmission geometry

5.5.5 Deviation parameter, extinction distance, penetration depth and Darwin width in the reflection geometry

5.5.6 Index of refraction for dynamical diffraction 135

5.6 The deviation parameter in absorbing crystals 136

5.7 Amplitude ratio of the refracted and reflected waves 136

5.7.1 Phase of the amplitude ratio in the transmission geometry

5.7.2 Phase of the amplitude ratio in the reflection geometry

5.8 Anomalous absorption

5.8.1 Effective absorption coefficient in the transmission geometry

5.8.2 Absorption coefficient in the propagation direction

5.8.3 Discussion of anomalous absorption-properties of the standing wavefield

5.8.4 Anomalous absorption in the reflection geometry-penetration depth

5.9 Dispersion surface when the Bragg angle is close to $\pi / 2 \quad 148$

5.9.1 Deviation from Bragg's angle and Darwin width 148

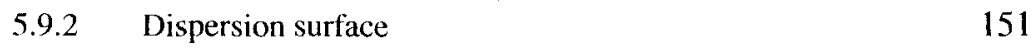

5.9.3 Penetration depth 153

$\begin{array}{lll}\text { 5.9.4 Applications } & 154\end{array}$

6 Intensities of plane waves in the transmission geometry

6.1 Boundary conditions for the amplitudes at the entrance surface

6.2 Amplitudes of the refracted and reflected waves 
6.3 Boundary conditions for the wavevectors at the exit surface

6.3.1 Condition for the existence of two outgoing waves

6.3.2 Wavevectors of the outgoing waves (Laue-Laue geometry)

6.3.3 Laue-Bragg geometry

6.4 Rocking curves of the reflected and refracted beams

6.4.1 Boundary conditions for the amplitudes at the exit surface

6.4.2 Reflectivity

6.4.3 Properties of the rocking curves

6.5 Integrated intensity

7 Intensities of plane waves in the reflection geometry

7.1 Thick absorbing crystals

7.1.1 Reflectivity

7.1.2 Shape of the rocking curves

7.2 Standing waves

7.3 Thin crystals

7.3.1 Boundary conditions for the amplitudes

7.3.2 Reflectivity

8 Dynamical diffraction in highly asymmetric coplanar and non-coplanar geometries

8.1 Introduction

8.2 Diffraction at grazing incidence or grazing emergence

8.3 Deviation from Bragg's incidence of the middle of the reflection domain

8.3.1 Grazing incidence and Bragg geometry

8.3.2 Grazing incidence, Laue geometry

8.3.3 Grazing emergence

8.4 Variation of the Darwin width for a grazing incidence

8.5 Variation of the width of the diffracted beam for a grazing emergence

$\begin{array}{ll}8.6 & \text { Equation of the dispersion surface } \\ 8.7 & 201\end{array}$

8.7 Relation with the traditional dynamical theory 206

$\begin{array}{lll}8.8 & \text { Specularly and Bragg-reflected intensities } & 207\end{array}$

8.8.1 Boundary conditions for the amplitudes at the entrance surface

8.8.2 Specularly and Bragg-reflected intensities for a grazing incidence and the Bragg geometry (semi-infinite crystal)

8.8.3 Specularly and Bragg-reflected intensities for a grazing incidence and the Laue geometry 

8.9.1 Introduction
8.9.2 Three-dimensional representation of the dispersion surface

8.9.3 Tiepoints excited by the incident wave

8.9.4 Equation of the dispersion surface

8.9.5 Amplitudes of the waves

$9 \quad n$-beam dynamical diffraction

9.1 Introduction 225

$\begin{array}{ll}9.2 & \text { The general three-beam case } \\ 9.226\end{array}$

9.2.1 Renninger-scans 226

9.2.2 Fundamental equations of the dynamical theory 227

9.2.3 Solution in the general case 233

9.2.4 Energy flow 235

9.3 The three-beam coplanar case $\quad 236$

9.4 Determination of phases using $n$-beam diffraction 236

$\begin{array}{ll}9.5 & \text { The super-Borrmann effect } \\ 9.52\end{array}$

9.5.1 Experimental evidence 242

9.5.2 Solution of the $111, \overline{1} 11$ case 243

9.5.3 Anomalous absorption coefficient 246

10 Spherical-wave dynamical theory: I. Kato's theory 249

10.1 Extension of the dynamical theory to any kind of incident wave 249

10.2 Fourier expansion of a spherical wave in plane waves 250

10.2.1 Principle of Kato's spherical-wave theory 250

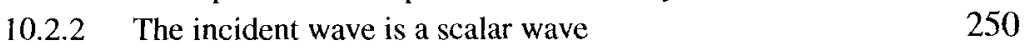

10.2.3 The incident wave is a vector wave 253

10.3 Direct integration in the transmission geometry 255

10.3.1 The geometrical conditions 255

10.3.2 Stationary phase method 257

10.3.3 Amplitude distribution on the exit surface-reflected
wave

10.3.4 Amplitude distribution on the exit surface-refracted wave $\quad 260$

10.4 Intensity distribution on the exit surface 260

10.5 Equal-intensity (Pendellösung) fringes 263

10.6 Integration by the stationary phase method 264

$\begin{array}{lll}10.7 & \text { Integrated intensity } & 268\end{array}$

$\begin{array}{ll}10.8 \text { Influence of polarization } & 269\end{array}$

$\begin{array}{lll}10.9 & \text { Bragg geometry } & 269\end{array}$

$\begin{array}{ll}10.10 & \text { Diffraction of ultrashort pulses } \\ & 274\end{array}$

Appendix: Geometrical interpretation of $\eta / \sqrt{S\left(\gamma_{h}\right)+\eta^{2}}$ in the transmission geometry 
11 Spherical-wave dynamical theory: II. Takagi's theory

11.1 Introduction

11.2 Generalized fundamental equations

11.2.1 Modulated waves

11.2.2 Takagi's equations

11.2.3 Boundary conditions for the amplitudes at the entrance surface

11.3 Reduction of Takagi's equations in the plane-wave case

11.4 Absorbing crystals

11.5 Analytical resolution of Takagi's equations for perfect crystals

11.6 Analytical solution for a point source using the method of integral equations

11.6.1 Transmission geometry

11.6.2 Reflection geometry

11.7 Analytical resolution of Takagi's equations using the Riemann function

11.7.1 Hyperbolic nature of Takagi's equations

11.7.2 General expression of the reflected and refracted waves

11.7.3 Determination of the Riemann function

11.7.4 General solution of Takagi's equations

11.8 Analytical solution for an incident spherical wave using the method of Riemann functions

11.8.1 The incident wave is a point source located on the entrance surface

11.8.2 The incident wave is a point source located away from the entrance surface

11.8.3 Conservation of energy

Appendix: Hyperbolic partial differential equations

Characteristics

Adjoint differential expression

12 Ray tracing in perfect crystals

$\begin{array}{lll}12.1 & \text { Ray tracing } & 304\end{array}$

12.2 The structure of real waves 305

12.3 Wavepackets made of the superposition of separate plane waves

12.4 Wavepackets made of a continuous distribution of wavevectors

$\begin{array}{ll}12.5 \text { Group velocity and Poynting vector } & 310\end{array}$

12.6 Angular amplification

12.7 Intensity distribution along the base of the Borrmann triangle (transmission geometry) 
12.8 Geometrical properties of wavefield trajectories within the Borrmann triangle

12.8.1 Wavefields propagating along the median, $\boldsymbol{A E}$, of the Borrmann triangle

12.8.2 Properties of the trajectories of the two wavefields excited by a plane wave

12.9 Experimental proof of double refraction

12.10 Experimental observation of the separation of the wavefield paths

12.10.1 Experimental setup $\quad 326$

12.10.2 Focalization of the various wavelengths 328

12.10.3 Separation of wavefield paths in the transmission case 329

12.10.4 Plane-wave Pendellösung $\quad 330$

12.10.5 Application to the measurement of the index of refraction

12.11 Fresnel diffraction near the Bragg incidence 335

12.12 Ray tracing in finite crystals $\quad 339$

12.12.1 Introduction 339

12.12.2 Bragg-Laue geometry-pseudo-plane waves 341

12.12.3 Bragg-Bragg geometry; multiple reflections of a pseudo-plane wave in thin crystals 343

12.12.4 Laue-Bragg geometry-Borrmann-Lehmann fringes $\quad 344$

12.13 Coherence of extended, non-strictly monochromatic sources $\quad 349$

\section{Extension of the dynamical theory to} deformed crystals

13 Ray tracing in slightly deformed crystals 355

13.1 X-ray propagation in deformed materials 355

13.1.1 The different degrees of deformation 355

13.1.2 Principle of ray theories for weak deformations 356

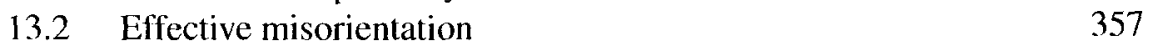

13.2.1 Local reciprocal lattice vector 357

13.2.2 Effective misorientation in direct space 359

13.2.3 Effective misorientation in reciprocal space $\quad 360$

13.2.4 Strain gradient $\quad 362$

13.3 Polarizability of a deformed crystal 363

13.4 The Eikonal approximation $\quad 363$

13.4.1 Justification of the concept of local dispersion surface $\quad 363$

13.4.2 Fermat's principle $\quad 365$

$\begin{array}{lll}\text { 13.5 Ray trajectories } & 368\end{array}$

$\begin{array}{ll}\text { 13.5.1 Local dispersion surface } & 368\end{array}$

$\begin{array}{ll}\text { 13.5.2 Local wavevectors } & 369\end{array}$

13.5.3 Differential equation of the wavefield trajectories $\quad 369$ 
13.6 The case of a constant strain gradient

13.6.1 Equation of the ray trajectory with respect to the lattice planes

13.6.2 Ray trajectories in the transmission geometry

13.6.3 Pure bending

13.6.4 Temperature gradient

13.6.5 Ray trajectories in the reflection geometry

13.7 Diffracted intensities-plane-wave case

13.7.1 Zero absorption

13.7.2 Absorbing crystals (transmission geometry)

13.7.3 Expression of the diffracted intensities for a constant strain gradient

13.7.4 Discussion of the intensity distribution for a constant strain gradient

13.8 Diffracted intensities-spherical-wave case

13.8.1 Pendellösung in slightly deformed crystals

13.8.2 Phase of the refracted wave in a deformed crystal

13.8.3 Expression of the phase in terms of the coordinates in direct space

13.8.4 Shape of the Pendellösung fringes in a deformed crystal

13.9 Lameller model

14 Propagation of X-rays in highly deformed crystals

14.2 Takagi's equations in a deformed crystal

14.3 Resolution of Takagi's equations in the deformed crystal case

14.3.1 Small deformations, limit of the validity of the

Eikonal approximation

14.3.2 Analytical resolution of Takagi's equations

14.3.3 Numerical integration

14.3.4 Applications

14.4 Ray concept applied to highly distorted crystals

14.4.1 Generalization of the notion of wavefields, interbranch scattering

14.4.2 Example: $\mathrm{X}$-ray propagation in a crystal with a concentration gradient (Keitel et al. 1999)

14.5 Statistical dynamical theories

14.5.1 Introduction

14.5.2 Principle of Kato's statistical dynamical theory

14.5.3 Experimental tests of the statistical dynamical theory

Appendix: Resolution of Takagi's equations in the case of a constant strain gradient using Laplace transforms 
IV Applications

15 X-ray optics

15.1 X-ray sources

15.1.1 X-ray tubes

15.1.2 Synchrotron radiation

15.2 Flat monochromators

15.2.1 Introduction

445

15.2.2 Monochromator crystals

446

15.2.3 Multiple-reflection monochromators

449

15.3 Applications of multiple-crystal arrangements to beam conditioning

15.3.1 Suppression of tails

456

15.3.2 Wavelength scanner

459

15.3.3 Production of beams with a very narrow angular spread

15.3.4 Harmonic suppression

461

15.3.5 Production of polarized radiation

15.4 Focusing optics

473

15.4.1 Introduction

473

15.4.2 Mirrors

474

15.4.3 Multilayers

476

15.4.4 Curved crystals

477

15.4.5 Fresnel zone plates

478

15.4.6 Bragg-Fresnel lenses

480

15.4.7 Refractive lenses

481

15.4.8 X-ray wave-guides

482

$15.5 \mathrm{X}$-ray interferometers

483

15.5.1 Principle

483

15.5.2 Applications 485

15.6 Imaging with X-rays 489

15.6.1 Introduction 489

15.6.2 Phase contrast imaging 489

16 Location of atoms at surfaces and interfaces using X-ray standing waves

16.1 Principle

16.2 Theory

16.2.1 Fluorescence yield

16.2.2 Influence of thermal vibrations

16.3 Bulk crystals

16.3.1 Extinction effect

16.3.2 Determination of the polarity of heteropolar crystals 
16.5 Thin films and buried interfaces

16.5.1 Simple model 507

16.5.2 Calculation of the standing pattern in an overlayer with the dynamical theory

16.6 Standing waves in deformed crystals

16.7 Standing waves due to specular reflection

17 X-ray diffraction topography

17.1 Introduction

17.2 Single-crystal reflection topography (Berg-Barrett technique)

17.2.1 Principle

17.2.2 Image formation

17.2.3 Penetration depth

17.2.4 Stereographic views

17.2.5 Applications

17.3 Single-crystal transmission topography

17.3.1 Early history

17.3.2 Principle of section topographs

523

17.3.3 Projection topographs

17.3.4 Dislocation images

17.3.5 Images of planar defects

17.3.6 Applications

17.4 Double- or multiple-crystal topography

17.4.1 Principle of double-crystal topography

17.4.2 Plane-wave crystal topography

17.4.3 Synchrotron double-crystal topography

17.4.4 Mapping of distortions and of lattice parameter variations

17.4.5 Equal-strain or equal-lattice parameter contours

17.4.6 Double-crystal setting for high spatial resolution topography

\section{Appendices}

Appendix 1 Useful formulae

Appendix 2 The early days of dynamical theory

List of symbols 